\title{
RATIONAL NORMAL MATRICES SATISFYING THE INCIDENCE EQUATION
}

\author{
DAVID HERTZIG
}

1. Introduction. The construction of a finite projective plane with $n$ points on a line is equivalent to determining an integral $v \times v$ matrix $A\left(v=n^{2}+n+1\right)$ satisfying

$$
A^{t} A={ }^{t} A A=n I+S
$$

where $S$ is the matrix all of whose coordinates are 1. The BruckRyser Theorem [2] asserts that when $n \equiv 1$ or $2(\bmod 4)$, a necessary and sufficient condition for the existence of a rational $A$ satisfying (1) is that $n$ be a sum of two squares. In [1] Albert gives a construction for such a rational $A$. The purpose of this note is to give a simpler construction.

2. Notation. We denote $I_{r}$ the $r \times r$ identity matrix, $S_{r}$ the $r \times r$ matrix all of whose entries are $1, e_{r}$ the $1 \times r$ matrix (row vector) all of whose entries are 1 . If $m=\frac{1}{2}(v-1)=\frac{1}{2} n(n+1)$, we denote

$$
E=\left(\begin{array}{cc}
0 & I_{m} \\
-I_{m} & 0
\end{array}\right)
$$

so that $E^{t} E=I_{2 m}$ and $E+{ }^{t} E=0$. We suppose $n=a^{2}+b^{2}$ and let $H=a I_{2 m}+b E$ so that $H^{t} H=n I_{2 m}$. Let

$$
P=\left(\begin{array}{cc}
0 & \frac{1}{n} e_{2 m} H \\
{ }^{e_{2 m}} & H
\end{array}\right) ;
$$

then clearly $P$ is a rational $v \times v$ matrix satisfying $P^{t} P=n I_{v}+S_{v}$. Moreover

$$
{ }^{t} P P=n I_{v}+{ }^{t} x x \text { where } \quad x=\left(n \frac{1}{n} e_{2 m} H\right)
$$

is a $1 \times v$ row vector.

3. A rational solution. To obtain a rational solution of (1) it suffices to find a rational orthogonal $v \times v$ matrix $T$ such that if $A=P T$ then ${ }^{t} A A=n I_{v}+S_{v}$. But ${ }^{t} A A={ }^{t} T^{t} P P T={ }^{t} T\left(n I_{v}+{ }^{t} x x\right) T=n I_{v}+{ }^{t}(x T)(x T)$;

Received by the editors July 30,1962 . 
hence it suffices to find a rational orthogonal $T$ satisfying ${ }^{t}(x T)(x T)$ $=S_{v}$. Since $S_{v}={ }^{\prime} b_{v} b_{v}$, we must find a rational $T$ satisfying $x T=e_{v}$. Noting that $x^{t} x=e_{v}{ }^{t} e_{v}=v$, i.e., the vectors $x$ and $b_{v}$ have the same length, it is apparent that the symmetry with respect to the plane perpendicular to the vector $x-e_{v}$ is the required transformation:

$$
T=I_{v}-\frac{2^{t}(x-e)(x-e)}{(x-e)^{t}(x-e)} .
$$

\section{REFERENCES}

1. A. A. Albert, Rational normal matrices satisfying the incidence equation, Proc. Amer. Math. Soc. 4 (1953), 554-559.

2. R. H. Bruck and H. J. Ryser, The nonexistence of certain finite prajective planes, Canad. J. Math. 1 (1949), 88-93.

Institute for Defense Analyses 